\title{
Adding high-intensity interval training to conventional training modalities: optimizing health-related outcomes during chemotherapy for breast cancer: the OptiTrain randomized controlled trial
}

\author{
Sara Mijwel ${ }^{1,2}$ (D) Malin Backman ${ }^{2,9} \cdot$ Kate A. Bolam $^{2,3} \cdot$ Anna Jervaeus $^{2} \cdot$ Carl Johan Sundberg $^{1,4} \cdot$ Sara Margolin $^{5,6}$. \\ Maria Browall $^{2,7} \cdot$ Helene Rundqvist $^{8} \cdot$ Yvonne Wengström $^{2,9}$
}

Received: 27 October 2017 / Accepted: 4 November 2017 / Published online: 14 November 2017

(c) The Author(s) 2017. This article is an open access publication

\begin{abstract}
Purpose Exercise training is an effective and safe way to counteract cancer-related fatigue (CRF) and to improve healthrelated quality of life (HRQoL). High-intensity interval training has proven beneficial for the health of clinical populations. The aim of this randomized controlled trial was to compare the effects of resistance and high-intensity interval training (RT-HIIT), and moderate-intensity aerobic and high-intensity interval training (AT-HIIT) to usual care (UC) in women with breast cancer undergoing chemotherapy. The primary endpoint was CRF and the secondary endpoints were HRQoL and cancer treatment-related symptoms.

Methods Two hundred and forty women planned to undergo chemotherapy were randomized to supervised RT-HIIT, AT-HIIT, or UC. Measurements were performed at baseline and at 16 weeks. Questionnaires included Piper Fatigue Scale, EORTC-QLQ-C30, and Memorial Symptom Assessment Scale.

Results The RT-HIIT group was superior to UC for CRF: total CRF $(p=0.02)$, behavior/daily life $(p=0.01)$, and sensory/ physical ( $p=0.03$ ) CRF. Role functioning significantly improved while cognitive functioning was unchanged for RT-HIIT compared to declines shown in the UC group $(p=0.04)$. AT-HIIT significantly improved emotional functioning versus $\mathrm{UC}(p=0.01)$ and was superior to UC for pain symptoms $(p=0.03)$. RT-HIIT reported a reduced symptom burden, while AT-HIIT remained stable compared to deteriorations shown by UC $(p<0.01)$. Only RT-HIIT was superior to UC for total symptoms $(p<0.01)$.

Conclusions 16 weeks of resistance and HIIT was effective in preventing increases in CRF and in reducing symptom burden for patients during chemotherapy for breast cancer. These findings add to a growing body of evidence supporting the inclusion of structured exercise prescriptions, including HIIT, as a vital component of cancer rehabilitation.
\end{abstract}

Trial registration Clinicaltrials.gov Registration Number: NCT02522260.

Keywords High-intensity interval training · Concurrent training · Breast cancer · Chemotherapy · Symptom burden . Health-related quality of life

Sara Mijwel

Sara.Mijwel@ki.se

1 Department of Physiology and Pharmacology, Karolinska Institutet, Stockholm, Sweden

2 Department of Neurobiology, Care Sciences and Society, Karolinska Institutet, Huddinge, Sweden

3 School of Human Movement and Nutrition Sciences, The University of Queensland, Brisbane, Australia

4 Department of Learning, Informatics, Management and Ethics, Karolinska Institutet, Stockholm, Sweden
5 Department of Clinical Science and Education, Södersjukhuset, Karolinska Institutet, Stockholm, Sweden

6 Department of Oncology, Stockholm South General Hospital, Stockholm, Sweden

7 School of Health and Education, University of Skövde, Skövde, Sweden

8 Department of Cell and Molecular Biology, Karolinska Institutet, Stockholm, Sweden

9 Theme Cancer, Karolinska University Hospital, Stockholm, Sweden 


\section{Introduction}

Adjuvant chemotherapy improves survival and decreases the risk of recurrence of breast cancer [1]. However, the treatment is associated with considerable side effects that negatively impact an individual's health-related quality of life (HRQoL) [2-4]. Cancer-related fatigue (CRF) is often reported as the most debilitating of all symptoms experienced during chemotherapy $[4,5]$, and has been described as a cancer treatment toxicity that can last for several years into survivorship [6].

Exercise training has been shown to improve HRQoL [7] and has been established as an effective intervention to manage CRF [8], being superior to both pharmacological and psychological interventions [9]. While both aerobic exercise and resistance exercise alone have shown positive health effects for women with breast cancer [10], cancerspecific exercise guidelines recommend that people with breast cancer engage in a combination of aerobic and resistance exercise [11].

There is increasing evidence highlighting the benefits of shorter bursts of high-intensity exercise for clinical populations $[12,13]$. The evidence shows not only improvements in cardiorespiratory fitness compared to moderate-intensity aerobic training, but also added benefits on quality of life [14], mood state [15, 16], and cognitive health [17] and increases endorphin release in brain areas associated with controlling emotion and pain [18]. Pilot studies have shown that patients with cancer can perform HIIT safely [19-21]. Given that one major barrier to perform physical exercise is lack of time and motivation [22], HIIT during chemotherapy may be favorable for optimizing health outcomes. We hypothesize that combining HIIT with conventional exercise provide added benefits on CRF, HRQoL, and symptom burden. This is the first large RCT to incorporate this emerging training modality in patients with breast cancer during chemotherapy. The aim of this study was to compare the effects of two training interventions: 1) resistance and high-intensity interval training (RT-HIIT), and 2) moderate-intensity aerobic and high-intensity interval training (AT-HIIT), to a control group receiving usual care (UC) on CRF, HRQoL, and symptoms in women with breast cancer undergoing chemotherapy.

\section{Methods}

\section{Study design}

The OptiTrain randomized controlled trial (NCT02522260, www.clinicaltrials.gov) was an in-clinic randomized controlled exercise trial [23]. Participants were randomly allocated to either group 1: RT-HIIT, group 2: AT-HIIT, twice a week for 16 weeks, or group 3: control group receiving UC. The primary endpoint CRF and the secondary endpoints HRQoL and symptom/symptom burden were measured 1 week prior to the participants' second chemotherapy session and at 16 weeks. The intervention groups (RT-HIIT and AT-HIIT) commenced the exercise training 3 days after the second chemotherapy session. The rationale for performing baseline assessments after the first round of chemotherapy was the limited time to perform ECG and baseline testing between randomization and the first chemotherapy session.

\section{Setting and participants}

Recruitment took place at two different oncology clinics in Stockholm, Sweden from March 2013 to July 2016 and 628 patients were eligible and invited to participate in the study by the referring oncologists. Two hundred and forty women volunteered to participate in the study. Eligibility criteria were as follows: women (i) aged 18-70 years, (ii) diagnosed with I-IIIa stage breast cancer, and (iii) planned to receive adjuvant chemotherapy (consisting of anthracyclines, taxanes, or a combination of the two). Participants were excluded if they had advanced disease, heart or lung disease, cognitive dysfunction, or did not speak or understand the Swedish language. Interested participants answered a questionnaire about their cardiovascular health history [24] and underwent a resting echocardiogram before enrollment to rule out cardiac pathologies. Ethical approval was obtained from the Regional Ethical Review Board in Stockholm Sweden (Dnr 2012/1347-31/1, 2012/1347-31/2, 2013/7632-32, and 2014/408-32) and all participants gave written informed consent.

\section{Randomization and blinding}

The participants were randomly allocated to RT-HIIT, AT-HIIT, or UC, by the Clinical Studies Unit at Karolinska University Hospital, using a random assignment computer program at a 1:1:1 ratio blinded to the research team, prior to the first assessment. Participants, exercise supervisors, and outcome assessors were not blinded to group allocation.

\section{Exercise intervention}

The RT-HIIT and AT-HIIT groups undertook the exercise sessions in an exercise clinic twice weekly for 16 weeks. Exercise session duration was approximately $60 \mathrm{~min}$, supervised by an exercise physiologist or an oncology nurse [23]. The program was extended for participants with delays in chemotherapy (RT-HIIT, $n=8$, range: $15-35$ days; AT-HIT, $n=5$, range: $13-32$ days). All exercise sessions 
began with a 5-min warm up on a cycle ergometer or treadmill at a rating of perceived exertion (RPE) of 10-12 on the Borg scale [25], and ended with a 10-min cool down of dynamic muscle stretching. The RT-HIIT group performed both resistance and high-intensity interval aerobic exercise at each session. The resistance training component consisted of exercises targeting the major muscle groups using weight stack training equipment, participants' body mass, free weight dumbbells or barbells. Exercises included leg press, biceps curls, squat jumps, triceps extensions, lunges, bench press, sit-ups or Russian-weighted abdominal twist, shoulder press, and prone-lying back extensions. Participants performed two to three sets of 8-12 repetitions at an initial intensity of $70 \%$ of their estimated one repetition maximum (1-RM) using a prediction equation [26], progressing to $80 \%$ of $1-\mathrm{RM}$ when more than 12 repetitions could be performed. To ensure overload, new estimated 1-RM tests were performed when participants could lift more than 12 repetitions of their $80 \% 1-\mathrm{RM}$. The aerobic component of the RT-HIIT program consisted of $3 \times 3$-min bouts of highintensity interval aerobic exercise on a cycle ergometer at a rating of perceived exertion of 16-18 on the Borg scale interspersed with one min of low-intensity active recovery. The AT-HIIT group commenced with 20 min of moderateintensity, continuous aerobic exercise at an RPE of 13-15 on a cycle ergometer, elliptical ergometer, or treadmill. This was followed by the same high-intensity interval exercise training as RT-HIIT. The UC group was given written information about physical activity at the initiation of the intervention period about exercise recommendations for patients with cancer according to American College of Sports Medicine guidelines [11].

\section{Outcome measures}

All outcome assessments took place at baseline and at 16 weeks.

\section{Primary outcome}

CRF was self-assessed using the Swedish version of the 22-item Piper Fatigue Scale (PFS) which has been validated in the Swedish population [27], covering four dimensions of fatigue: behavioral/daily life (6 items), sensory/physical ( 5 items), cognitive (6 items), and affective/emotional meaning (5 items). Each item is composed of a scale from zero to ten, with zero indicating "no fatigue." Scores were calculated according to recommended scoring procedures [28]. The instrument is reliable and has been used extensively in patients with breast cancer even though sensitivity to change is yet to be established [27].

\section{Secondary outcomes}

The 30-item EORTC-QLQ-C30 questionnaire (version 3.0) was used to assess HRQoL [29]. This questionnaire incorporates a global HRQoL score, five functional subscales, and nine symptoms, including general CRF. Despite lacking different dimensions of CRF, the EORTC-QLQ-C30 is valid and reliable to assess CRF [30], and is sensitive to change when used for patients with cancer during chemotherapy [31]. Symptoms and symptom burden were assessed using the 32-item Memorial Symptom Assessment Scale (MSAS) $[32,33]$. The scale includes occurrence, frequency, severity, and distress associated with each symptom using four- and five-point rating scales. Total symptom score was calculated as the average of all 32 symptoms, and symptom burden (the degree to which the symptoms affect daily life) was calculated as the average of the frequency of four psychological symptoms and the distress associated with six physical symptoms. The physical and psychological symptom subscales were calculated as the average of the frequency, severity, and distress associated with 12 physical symptoms, and six psychological symptoms, respectively.

To explore if baselines values influenced change over the intervention, sub-analyses were performed for the outcomes CRF, global HRQoL, symptom burden, and total symptoms. Cut-off values at baseline were categorized as CRF (score $>0$ ) and low global HRQoL according to a validated cut-off score (score < 80) [34]. For symptoms, the cut-off was chosen based on participants' mean score at baseline: high symptom burden (score $>0.79$ ), and high total symptom (score $>0.66$ ). No statistics were performed for these variables.

\section{Additional measures}

Demographic and medical information were collected through questionnaires and extracted from patients' electronic medical records. Objectively measured activity patterns were assessed at baseline by an accelerometer (GT3X ActiGraph ${ }^{\circledR}$ Corp, Pensacola, Florida, USA), which the participants were instructed to wear on an elastic belt over their right hip during all waking hours for seven consecutive days. Data were downloaded using the ActiLife v.6.10.1 software and analyzed using validated wear-time specifications and cut-offs for adults [35].

Attendance was calculated as the mean of the individual percentages (attended exercise sessions divided by the total number of sessions). Adherence to the exercise regimen was calculated as the number of patients who successfully completed $90 \%$ of the exercise sessions according to plan (i.e., intensity and duration), divided by the total number of patients in the intervention groups. 


\section{Adverse events}

At each exercise session, participants reported any adverse events to the exercise supervisors and these were recorded in the participants' log records.

\section{Statistical methods}

With CRF as the primary outcome measure, a sample size of 65 participants per group was required, based on an effect size of 0.53 and $80 \%$ power at an alpha level of 0.05 (twotailed). With an expected attrition rate of $20 \%, 80$ participants were required per group. Data were analyzed using the IBM $^{\circledR}$ SPSS $^{\circledR}$ version 22 statistical package for Windows. Analyses included standard descriptive statistics, one-way analysis of variance (ANOVA), and analysis of covariance (ANCOVA), controlling for baseline scores, chemotherapy treatment (taxanes/no taxanes), and menopausal status. Where appropriate, the Bonferroni post hoc procedure for multiple comparisons was used to locate the source of significant differences. Data not normally distributed were logtransformed prior to ANCOVA analyses. RM-ANOVA or the Wilcoxon signed rank test was applied for within-group analyses (within-group results shown in Tables 2, 3, and 4). Clinically important changes were estimated as standardized effect sizes (ES) where between-group differences of pre- and post-intervention means were divided by the pooled baseline standard deviation [36]:

$\frac{\left(M_{\text {post.T }}-M_{\text {pre.T }}\right)-\left(M_{\text {post.C }}-M_{\text {pre.C }}\right)}{S D_{\text {pooled pre }}}$.

According to Cohen's guidelines, effect sizes with scores of $0.2-0.5,0.5-0.8$, and $>0.8$ were considered small, medium, and large effects, respectively [37]. An intentionto-treat analysis (ITT) approach was used for the analyses, with missing values from the post-assessment being replaced using the expectation maximization method [38]. Participants that dropped out after baseline assessment and did not complete questionnaires at baseline were excluded from the analysis $(n=2)$. The expectation-maximization algorithm was based on group change. All tests were two-tailed and statistical significance was set at $p<0.05$.

\section{Results}

In total, 240 women agreed to participate, and 182 participants completed the baseline and follow-up testing. Postassessment data were imputed for participants $(n=22)$ who had completed baseline assessment for all outcomes. The main reasons for declining to participate after randomization were too time consuming, feeling ill, or did not want to be randomized to the UC group. The CONSORT study flow chart is shown in Fig. 1. There were no significant differences at baseline between groups regarding participant characteristic data (Table 1). Immediately following randomization, fourteen participants dropped out, while ten percent of the participants dropped out during the intervention. There were no significant differences in participant characteristics between those who withdrew and participants who completed the study. Attendance rates for participants in the RT-HIIT and AT-HIIT groups were 68 and 63\%, respectively, and adherence to the training program was $83 \%$ in the RT-HIIT group and 75\% in AT-HIIT group.

\section{Changes is CRF}

Following the intervention, CRF assessed by the Piper Fatigue Scale increased significantly in the UC group and was significantly different from maintained levels found for RT-HIIT: total CRF (ES = - 0.51), behavior/daily life CRF $(\mathrm{ES}=-0.62)$, and sensory/physical CRF $(\mathrm{ES}=-0.47)$ (Table 2). Similarly, CRF assessed by the EORTC-QLQC30 showed a significant increase for UC that was significantly different from the unchanged CRF for RT-HIIT $(\mathrm{ES}=-0.61)$ and for AT-HIIT $(\mathrm{ES}=-0.47)$ (Table 3$)$.

\section{Changes in HRQoL}

HRQoL measured by the EORTC-QLQ-C30 instrument showed a significant difference in role functioning in favor of RT-HIIT (ES = 0.81) and AT-HIIT $(\mathrm{ES}=0.64)$ compared to declines for UC. Cognitive functioning was superior for RT-HIIT compared to declines reported by $\mathrm{UC}(\mathrm{ES}=0.35)$, while AT-HIIT significantly improved in emotional functioning compared to UC (ES $=0.40)$. The magnitude of negative change in physical functioning for RT-HIIT $(\mathrm{ES}=0.49)$ and AT-HIIT $(\mathrm{ES}=0.48)$ were significantly lower compared to UC. UC reported significantly higher pain scores compared to unchanged pain scores reported by AT-HIIT (ES = - 0.36) (Table 3).

\section{Changes in symptoms and symptom burden}

A significant difference was found for symptom burden as measured by the MSAS, with RT-HIIT (ES = - 0.43) reporting a reduced symptom burden score and AT-HIIT ( $\mathrm{ES}=-0.42$ ) was unchanged compared to an increased score for UC. The RT-HIIT group reported unchanged levels of total symptoms being significantly different compared to the increase in symptoms experienced by UC $(\mathrm{ES}=-0.52)$ (Table 4). 


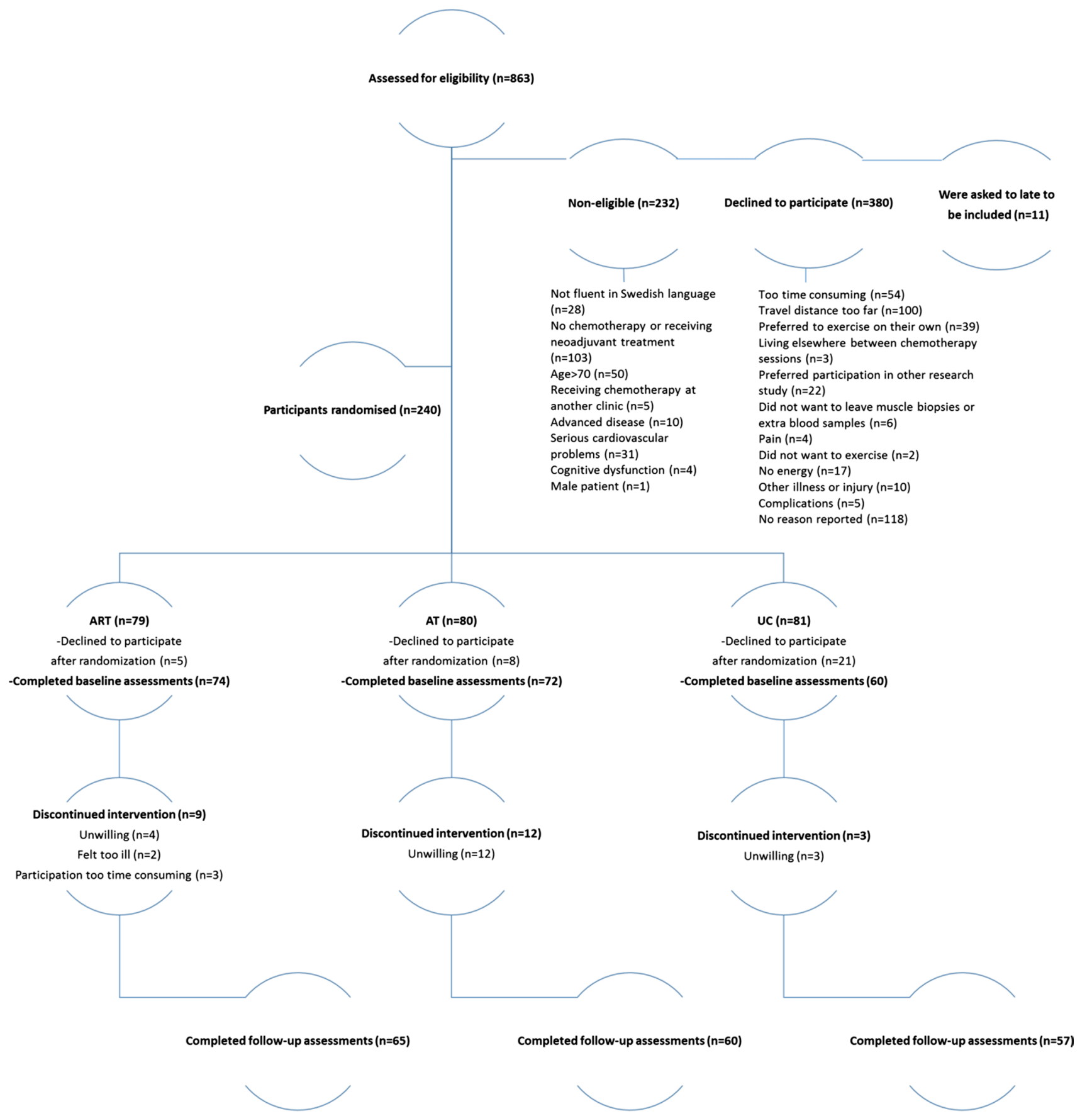

Fig. 1 CONSORT diagram. RT-HIIT resistance and high-intensity interval training, AT-HIIT moderate-intensity aerobic and high-intensity interval training, $U C$ usual care

\section{Baseline status and changes in CRF, HRQoL, and symptom burden}

Changes at 16 weeks for the outcomes CRF, global HRQoL, symptom burden, and total symptoms based on baseline status are shown in Fig. 2. In the sub-analysis, we show that, for the participants experiencing CRF at baseline, those in the UC group reported a further increase in CRF at the follow-up, while those in the exercise groups reported decreased levels of CRF. Similar results were shown for global HRQoL, symptom burden, and total symptom score where the participants exhibited beneficial effects from exercise training if they had reported a low global HRQoL or displayed a high symptom burden at baseline.

At baseline, objectively assessed physical activity was significantly higher in groups reporting no CRF compared 
Table 1 Participant characteristics at baseline (mean \pm SD)

\begin{tabular}{|c|c|c|c|}
\hline & $\begin{array}{l}\text { RT-HIIT } \\
n=74 \\
\text { Mean } \pm \text { SD }\end{array}$ & $\begin{array}{l}\text { AT-HIIT } \\
n=72 \\
\text { Mean } \pm \text { SD }\end{array}$ & $\begin{array}{l}\text { UC } \\
n=60 \\
\text { Mean } \pm \text { SD }\end{array}$ \\
\hline Age (years) & $52.7 \pm 10.3$ & $54.4 \pm 10.3$ & $52.6 \pm 10.2$ \\
\hline Body mass (kg) & $68.7 \pm 11.3$ & $67.7 \pm 13.0$ & $69.1 \pm 11.0$ \\
\hline Height & $165.7 \pm 6.7$ & $165.3 \pm 6.6$ & $166.4 \pm 7.0$ \\
\hline MVPA (min/day) & $79.0 \pm 25.0$ & $71.0 \pm 31.0$ & $68.0 \pm 31.0$ \\
\hline \multirow[t]{2}{*}{$\operatorname{SED}(\min /$ day $)$} & $530.0 \pm 70.0$ & $544.0 \pm 60.0$ & $548.0 \pm 67.0$ \\
\hline & $\mathrm{n}(\%)$ & $\mathrm{n}(\%)$ & $\mathrm{n}(\%)$ \\
\hline Married or partnered & 60.6 & 59.7 & 69.5 \\
\hline \multicolumn{4}{|l|}{ Education level completed } \\
\hline Primary school & 16.9 & 16.2 & 17.0 \\
\hline Secondary school & 15.5 & 19.1 & 17.0 \\
\hline Tertiary education & 67.6 & 64.7 & 66.0 \\
\hline Current smoker & 4.3 & 5.9 & 5.2 \\
\hline Employed & 74.6 & 86.8 & 79.7 \\
\hline \multicolumn{4}{|l|}{ Menopausal status } \\
\hline Premenopausal & 47.3 & 36.1 & 38.3 \\
\hline Postmenopausal & 51.4 & 63.9 & 61.7 \\
\hline Not known & 1.4 & 0.0 & 0.0 \\
\hline \multicolumn{4}{|l|}{ Tumor profile } \\
\hline Triple negative & 14.9 & 11.0 & 16.7 \\
\hline HER2+, ER+, PR+ & 16.2 & 15.1 & 5.0 \\
\hline HER2+, ER-, PR- & 0.0 & 11.0 & 10.0 \\
\hline HER2-, ER+, PR+ & 54.1 & 46.6 & 53.3 \\
\hline HER2-, ER+, PR- & 8.1 & 12.3 & 8.3 \\
\hline HER2+, ER+, PR- & 5.4 & 4.1 & 5.0 \\
\hline HER2-, ER-, PR+ & 1.4 & 0.0 & 1.7 \\
\hline \multicolumn{4}{|l|}{ Chemotherapy regimen } \\
\hline Anthracycline & 39.3 & 37.0 & 40.0 \\
\hline Taxane & 2.7 & 5.5 & 0.0 \\
\hline Anthracycline + taxane & 37.8 & 37.0 & 33.3 \\
\hline Anthracycline + taxane + herceptin & 18.9 & 20.6 & 25.0 \\
\hline Anthracycline + herceptin & 1.4 & 0.0 & 1.7 \\
\hline
\end{tabular}

$R T-H I I T$ resistance and high-intensity interval training, $A T-H I I T$ moderate-intensity aerobic and high-intensity interval training, $U C$ usual care, $S D$ standard deviation, MVPA objectively measured moderate- to vigorous-intensity physical activity, $S E D$ objectively measured sedentary behavior

to those experiencing CRF ( $p=0.02)$, with high global HRQoL levels compared to low global HRQoL at baseline $(p=0.01)$ and with a low total symptom score compared to high total symptom score at baseline $(p=0.04)$ (Fig. 3).

No adverse events were reported as a result of the testing or the exercise sessions. Of particular clinical importance, no adverse events were reported in relation to peripherally inserted central catheters (inserted in the majority of the participants' arms) during the study period.

\section{Discussion}

In the current trial, the effects of RT-HIIT, and aerobic and high-intensity interval training (AT-HIIT) compared to UC were assessed in women with breast cancer undergoing chemotherapy. We show that 16 weeks of RT-HIIT counteracts several dimensions of CRF and reduces symptom burden. Both RT-HIIT and AT-HIIT groups showed beneficial effects for vital aspects of HRQoL. 


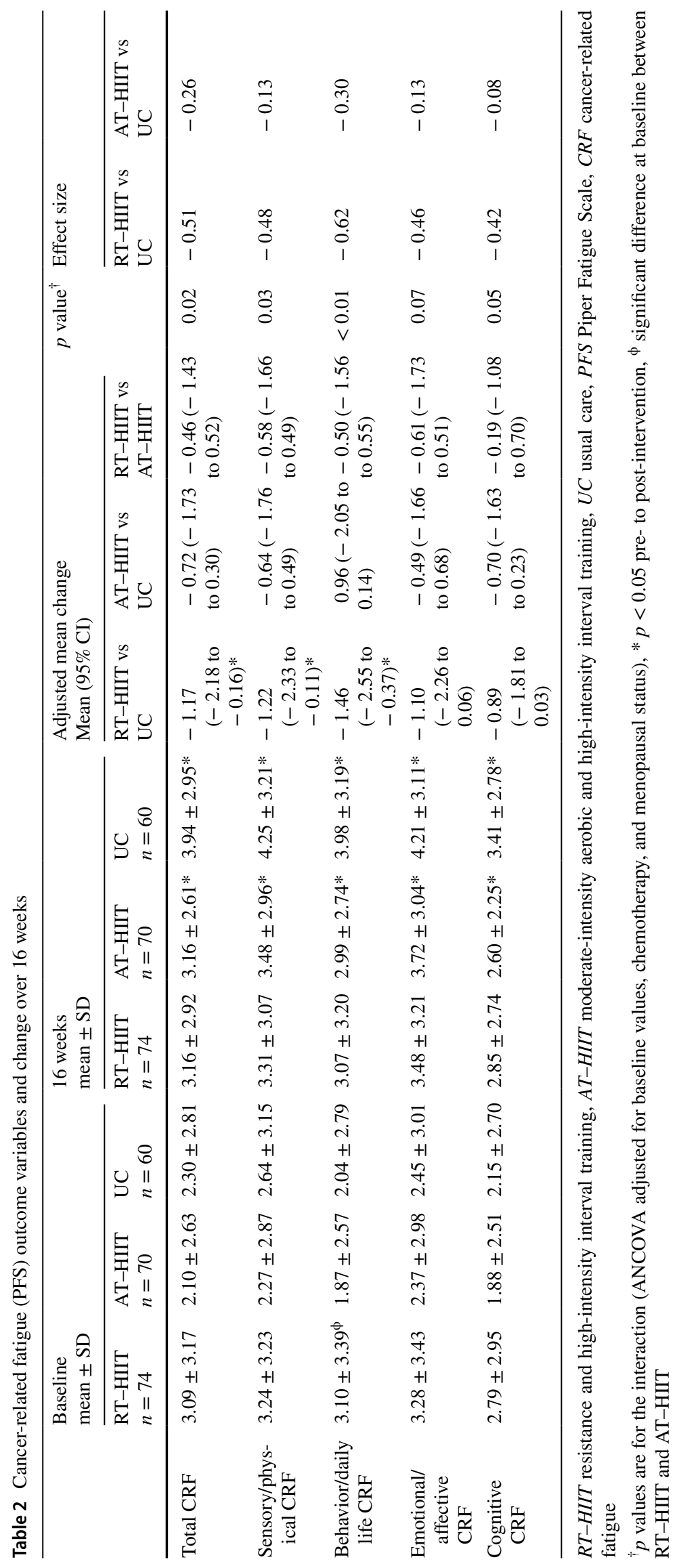




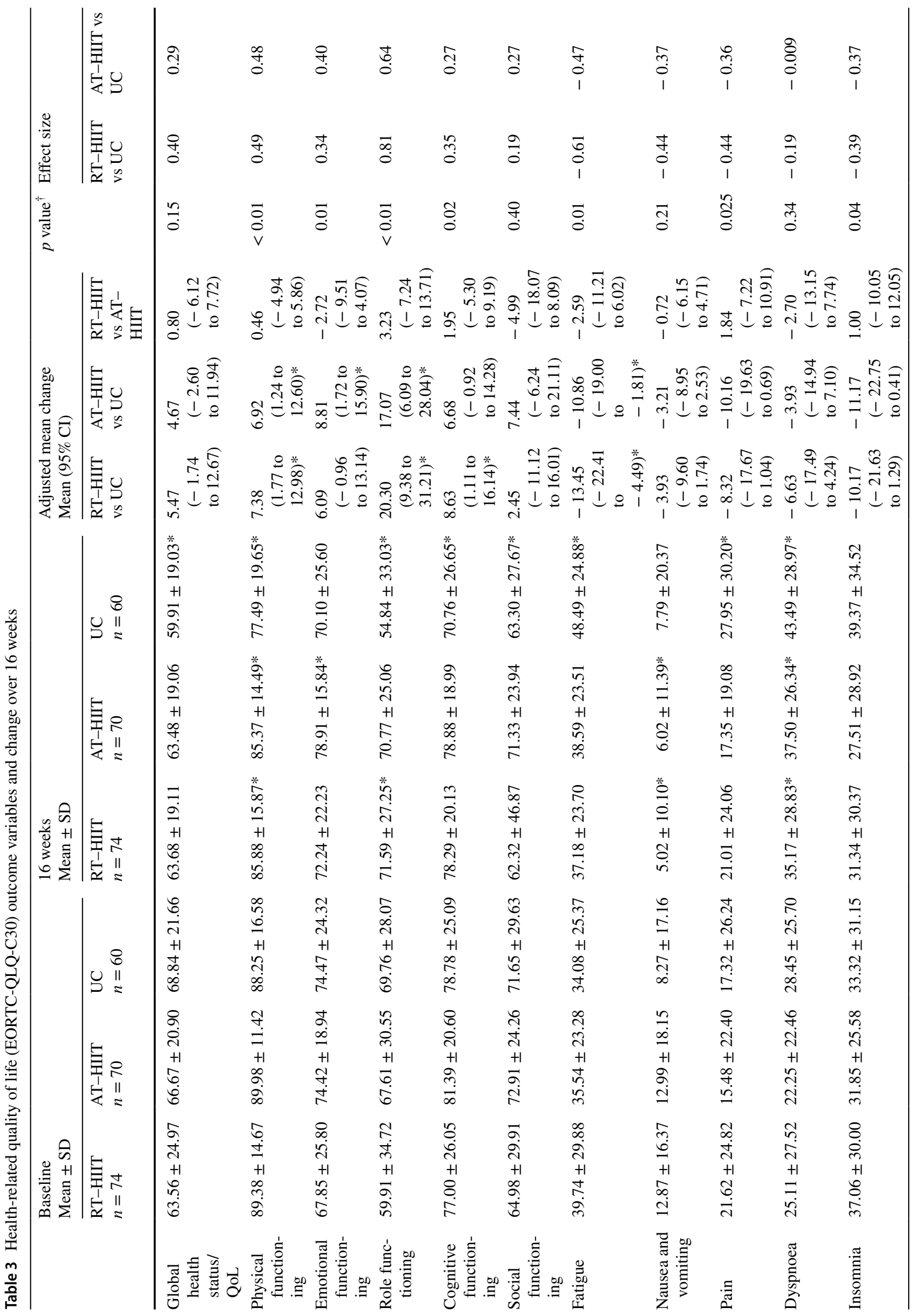




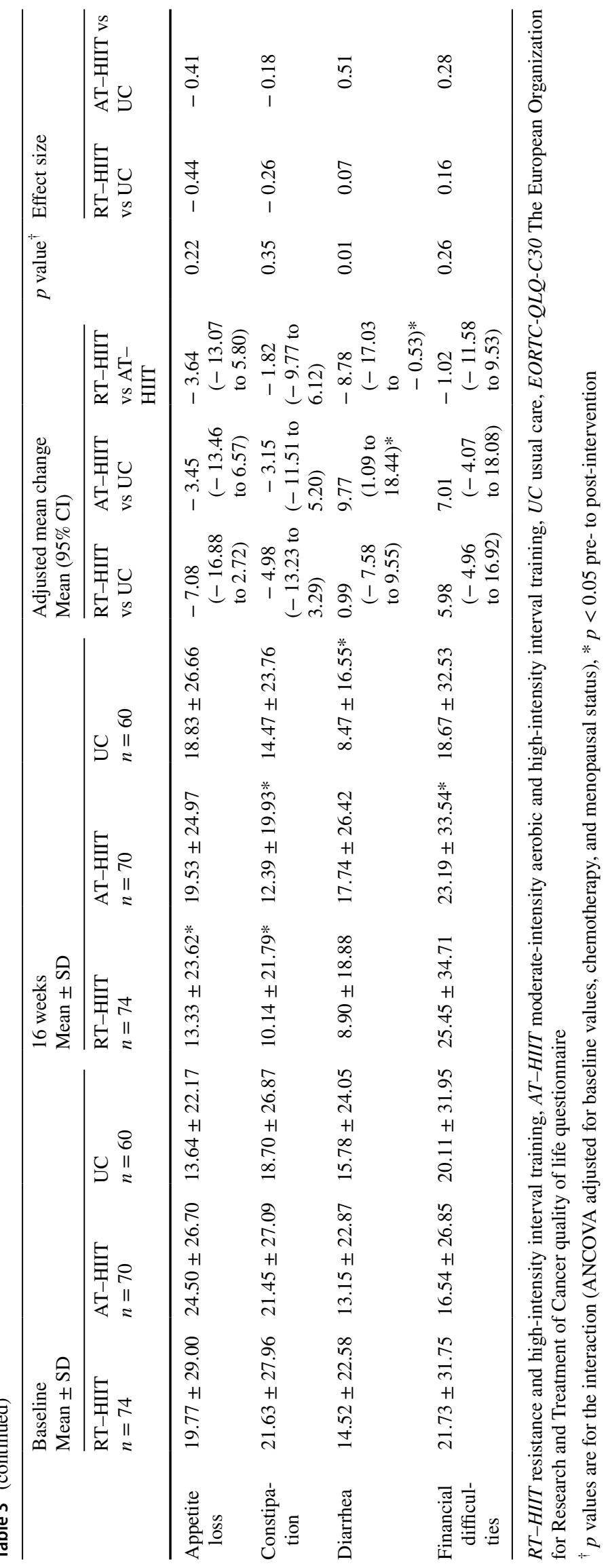




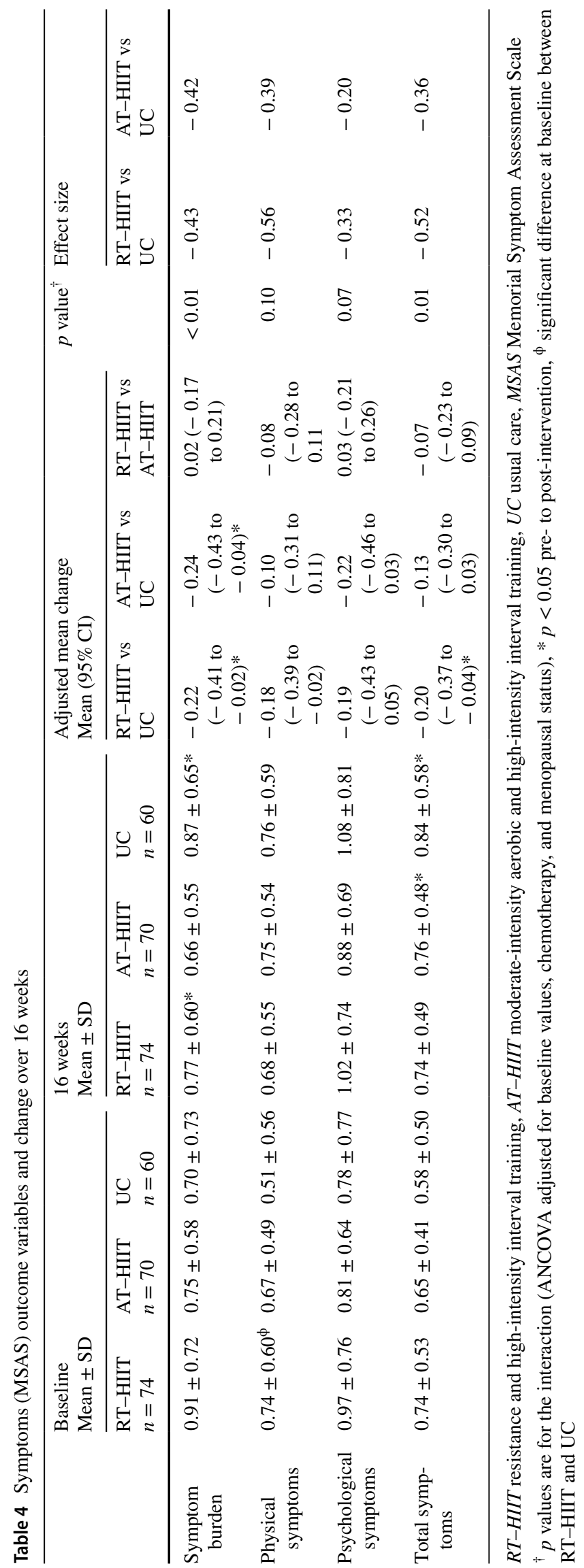


Fig. 2 Baseline status and change after 16 weeks for the outcomes. a CRF (assessed by the Piper fatigue scale), b global HRQoL (assessed by the European Organization for Research and Treatment of cancer quality of life questionnaire), c symptom burden (assessed by the Memorial symptom assessment scale), and $\mathbf{d}$ total symptom score (assessed by the Memorial symptom assessment scale). $C R F$ cancer-related fatigue, $H R Q o L$ health-related quality of life, $C R F$ cancer-related fatigue, $H R Q o L$ health-related quality of life, $R T-H I I T$ resistance and high-intensity interval training, $A T-H I I T$ moderate-intensity aerobic and high-intensity interval training, $U C$ usual care
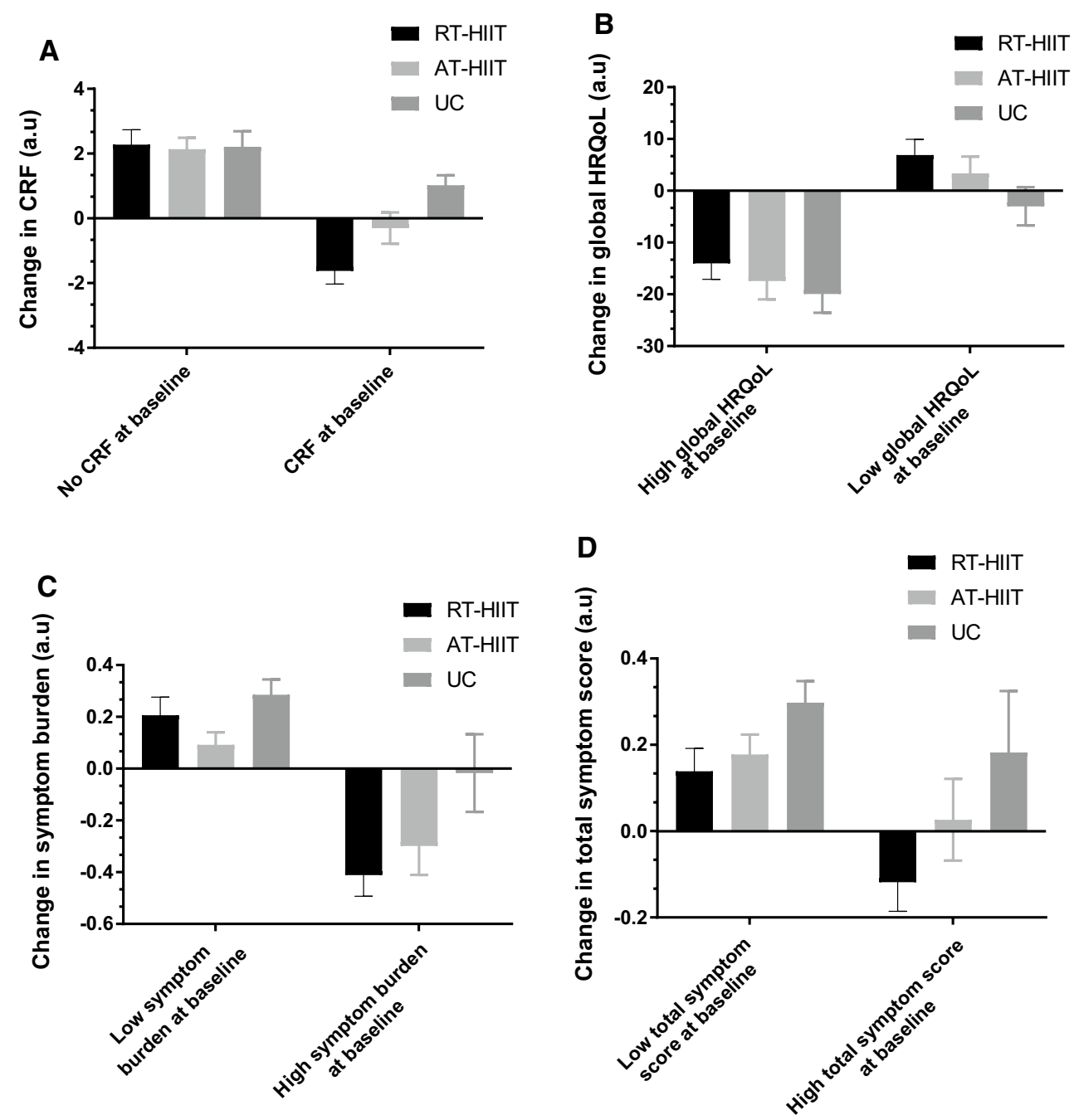

RT-HIIT counteracted increases in total CRF $(\mathrm{ES}=-0.51)$, including physical $(\mathrm{ES}=-0.48)$ and behavior/daily life aspects (ES $=-0.62$ ) of CRF. However, no effects for cognitive or emotional CRF were found. This is in line with a recent meta-analysis [5] showing that not all dimensions of fatigue are affected by exercise training. Similar to our findings, a recent trial [39], including moderate- to high-intensity aerobic exercise and progressive resistance exercise, demonstrated that concurrent resistance and aerobic exercise training was effective in offsetting CRF, with an effect size of -0.63 for physical CRF and -0.29 for general CRF. Superior effects of moderate/high intensity was also shown compared to lower intensity, home-based exercise for physical CRF (ES $=0.42$ ). In contrast, other trials $[10,40,41]$ showed no difference in CRF between participants performing moderate-intensity combined aerobic and resistance training versus a control group, suggesting that higher intensity/load combined exercise training is required to counteract increases in CRF. Noteworthy, in the present study, the AT-HIIT group was not able to counteract an increase in multi-dimensional measures of CRF following the intervention period. However, results from the unidimensional EORTC-QLQ-C30 scale showed that participants in the AT-HIIT group were able to counteract CRF, similar to effects displayed by the RT-HIIT group, although the effect size for RT-HIIT was higher (- 0.61) compared to AT-HIIT $(-0.47)$. Only one previous trial [42] incorporating multimodal training, which included high-intensity exercise training, has shown positive effects on CRF from the unidimensional EORTC-QLQ-C30 scale, leading us to, again, speculate that the beneficial and clinically relevant effects of exercise on CRF are intensity-dependent. The discrepant results for AT-HIIT when assessing multi-dimensional versus unidimensional fatigue are unclear. It may be that the PFS is not sensitive enough to detect clinically meaningful changes over time [27].

This is the first trial to demonstrate significant beneficial effects of exercise training on role and emotional functioning in patients with breast cancer during chemotherapy. The large effect size for the RT-HIIT group compared to UC for 

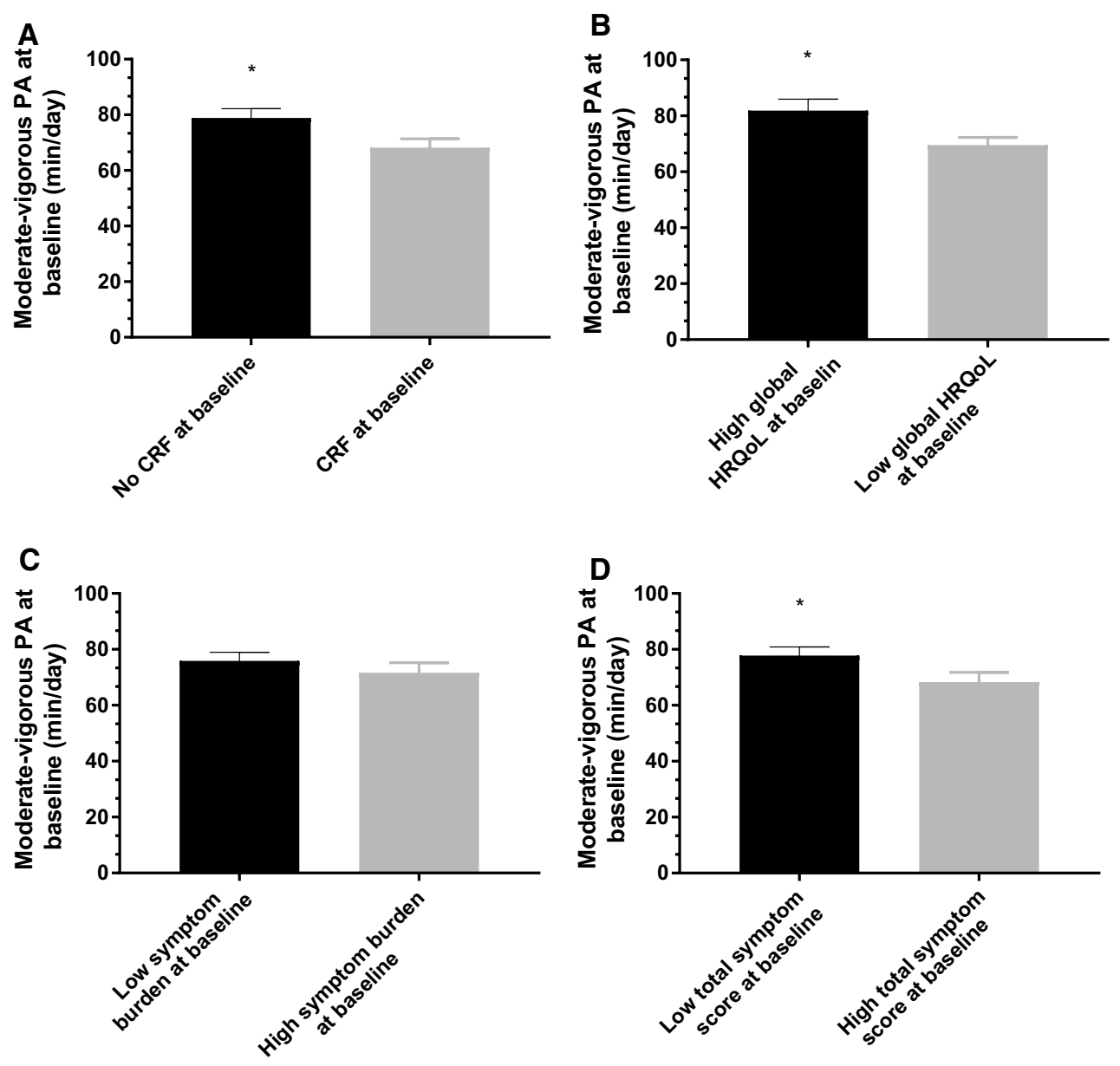

Fig. 3 Activity levels at baseline based on the subgroups. a CRF and no CRF at baseline (assessed by the Piper fatigue scale), b low global HRQoL and high global HRQoL at baseline (assessed by the European Organization for Research and Treatment of cancer quality of life questionnaire), $\mathbf{c}$ high symptom burden and low symptom burden

role functioning $(\mathrm{ES}=0.81)$ suggests an important role for the resistance training component in particular. It has been shown that being involved in a supervised exercise program can improve both role and emotional functioning [43], likely through interactions with supervising personnel and peers in the exercise setting. Notably, only AT-HIIT improved in emotional functioning. Aerobic training has been proven superior to resistance training alone in improving emotional functioning in elderly [44]. Moreover, aerobic exercise has been shown to decrease depressive and anxiety symptoms in breast cancer survivors [45]. An increased level of selfefficacy has been suggested as a possible mechanism for improved emotional functioning in elderly engaged in exercise [43], which in this case may be linked to feelings of being able to carry out the exercises and feeling more revitalized after the exercise sessions.

Results from the current trial demonstrated that RT-HIIT counteracted increases in self-reported cognitive problems.

at baseline (assessed by the Memorial symptom assessment scale), and $\mathbf{d}$ high total symptom score and low total symptom score at baseline (assessed by the Memorial symptom assessment scale). CRF cancer-related fatigue, $H R Q o L$ health-related quality of life. ${ }^{*} p<0.05$ between subgroups

The effect size of 0.35 is comparable to one previous trial that included high-intensity exercise, with a similar effect size (0.32) [39], while other trials [42, 46, 47] have failed to show beneficial effects of exercise on cognitive functioning. Once more, we speculate that intensity may play a crucial role in offsetting a lowered HRQoL. Cognitive dysfunction experienced during chemotherapy is associated with psychological distress and persists for several years post chemotherapy [48]. Pain is another debilitating symptom caused by chemotherapy. In the current trial, the AT-HIT group experienced significantly less pain compared to the UC group. A substantial number of patients receiving anthracycline/taxane-based chemotherapy experience pain, particularly during the taxane component [49] that is not relieved by analgesics [50]. Therefore, it is of great importance to implement successful interventions, such as high-intensity training, at an early stage in the survivorship continuum to aid in symptom relief. 
Our results demonstrate that exercise training during chemotherapy is effective to minimize the burden of symptoms related to chemotherapy. Few exercise trials have reported on symptom burden among women with breast cancer [45]. One consequence of a high degree of symptom burden may be chemotherapy dose reductions or even discontinued treatment, which in turn may influence prognosis [51]. In fact, two previous trials [10, 39] showed the potential of exercise to improve chemotherapy completion rates, and a trial by Courneya et al. [52] demonstrated that adding exercise to standard chemotherapy may improve breast cancer outcomes. With an increasing breast cancer survival rate, a larger number of women will experience different long-term side effects [2], including symptoms related to chemotherapy, which not only affects individuals' HRQoL negatively, but also aspects of daily life involving family and work performance [53]. Given the findings from this trial showing the effectiveness of exercise for managing symptom burden, it is of great importance to support patients to be physically active during treatment in order to prevent a progressive downward spiral in declining health for these patients.

In concordance with a recent trial [54], in a sub-analysis, we found that the participants with the highest levels of fatigue and symptoms and the lowest HRQoL were those who gained the greatest benefits from the exercise training. Importantly, participants with higher levels of CRF, symptoms, and lower HRQoL were also significantly less active than those with lower levels of CRF, symptoms, and a better HRQoL at baseline. It is therefore likely that beneficial effects from any exercise training regimens are dependent on fitness status at baseline.

Strengths of the OptiTrain trial are the RCT design, that two types of supervised exercise regimens were trialed during chemotherapy, the use of validated instruments, and a large sample size. Limitations of the study include the high dropout rate by the UC patients directly following randomization that may have led to a selection bias. This aspect should generally be considered when drawing conclusions from exercise intervention studies. While our recruitment and attendance rates are within the range commonly reported in exercise trials [55], supporting participants to complete exercise programs as prescribed remains a challenge for the field of exercise oncology.

\section{Conclusion}

In conclusion, results from the OptiTrain study show that a training program including RT-HIIT is effective to counteract both multi- and unidimensional aspects of CRF and to reduce symptom burden, in women with breast cancer during chemotherapy. Both RT-HIIT and AT-HIIT were effective to improve or maintain vital aspects of HRQoL. Findings from the OptiTrain trial add important evidence on benefits of high-intensity interval training for health outcomes for patients during chemotherapy, allowing health professionals in the oncology setting to recommend adding high-intensity interval exercise to specific and targeted programs for patients with breast cancer.

Acknowledgements The authors would like to thank the women who participated in this clinical intervention trial, the oncological rehabilitation unit at the University hospital in Stockholm (Sweden) for providing the exercise facilities, and Daniele Cardinale at the Swedish Sports Confederation (Lidingö, Sweden) for technical support.

Funding This work was supported by The Swedish Cancer Society (130452 to Y.W.), The Cancer Society of Stockholm (131242 to Y.W.), The Swedish Cancer and Traffic Accident Foundation (F-C-001225 to Y.W.), and The Swedish Society for Medical Research (SLS 50514 to H.R.).

\section{Compliance with ethical standards}

Availability of data and material The datasets used and/or analyzed during the current study are available from the corresponding author on reasonable request.

Conflict of interest The authors declare that they have no conflict of interest.

Ethical approval All procedures performed in studies involving human participants were in accordance with the ethical standards of the institutional and/or national research committee and with the 1964 Helsinki declaration and its later amendments or comparable ethical standards.

Open Access This article is distributed under the terms of the Creative Commons Attribution 4.0 International License (http://creativecommons.org/licenses/by/4.0/), which permits unrestricted use, distribution, and reproduction in any medium, provided you give appropriate credit to the original author(s) and the source, provide a link to the Creative Commons license, and indicate if changes were made.

\section{References}

1. Peto R, Davies C, Godwin J, Gray R, Pan HC, Clarke M, Cutter D, Darby S, McGale P, Taylor C, Wang YC, Bergh J, Di Leo A, Albain K, Swain S, Piccart M, Pritchard K (2012) Comparisons between different polychemotherapy regimens for early breast cancer: meta-analyses of long-term outcome among 100,000 women in 123 randomised trials. Lancet (Lond, Engl) 379(9814):432444. https://doi.org/10.1016/s0140-6736(11)61625-5

2. Barbaric M, Brooks E, Moore L, Cheifetz O (2010) Effects of physical activity on cancer survival: a systematic review. Physiother Can 62(1):25-34. https://doi.org/10.3138/physio.62.1.25

3. Davies NJ, Batehup L, Thomas R (2011) The role of diet and physical activity in breast, colorectal, and prostate cancer survivorship: a review of the literature. Br J Cancer 105(Suppl 1):S52S73. https://doi.org/10.1038/bjc.2011.423

4. Bower JE (2014) Cancer-related fatigue-mechanisms, risk factors, and treatments. Nat Rev Clin Oncol 11(10):597-609. https://doi. org/10.1038/nrclinonc.2014.127 
5. van Vulpen JK, Peeters PH, Velthuis MJ, van der Wall E, May AM (2016) Effects of physical exercise during adjuvant breast cancer treatment on physical and psychosocial dimensions of cancerrelated fatigue: a meta-analysis. Maturitas 85:104-111. https:// doi.org/10.1016/j.maturitas.2015.12.007

6. Medysky ME, Temesi J, Culos-Reed SN, Millet GY (2017) Exercise, sleep and cancer-related fatigue: are they related? Clin Neurophysiol 47(2):111-122. https://doi.org/10.1016/j. neucli.2017.03.001

7. Casla S, Lopez-Tarruella S, Jerez Y, Marquez-Rodas I, Galvao DA, Newton RU, Cubedo R, Calvo I, Sampedro J, Barakat R, Martin M (2015) Supervised physical exercise improves $\mathrm{VO}_{2 \max }$, quality of life, and health in early stage breast cancer patients: a randomized controlled trial. Breast Cancer Res Treat 153(2):371382. https://doi.org/10.1007/s10549-015-3541-x

8. Meneses-Echavez JF, Gonzalez-Jimenez E, Ramirez-Velez R (2015) Supervised exercise reduces cancer-related fatigue: a systematic review. J Physiother 61(1):3-9. https://doi.org/10.1016/j. jphys.2014.08.019

9. Mustian KM, Alfano CM, Heckler C et al (2017) Comparison of pharmaceutical, psychological, and exercise treatments for cancer-related fatigue: a meta-analysis. JAMA Oncol 3(7):961-968. https://doi.org/10.1001/jamaoncol.2016.6914

10. Courneya KS, Segal RJ, Mackey JR, Gelmon K, Reid RD, Friedenreich CM, Ladha AB, Proulx C, Vallance JK, Lane K, Yasui Y, McKenzie DC (2007) Effects of aerobic and resistance exercise in breast cancer patients receiving adjuvant chemotherapy: a multicenter randomized controlled trial. J Clin Oncol 25(28):4396-4404. https://doi.org/10.1200/jco.2006.08.2024

11. Schmitz KH, Courneya KS, Matthews C, Demark-Wahnefried W, Galvão DA, Pinto BM, Irwin ML, Wolin KY, Segal RJ, Lucia A, Schneider CM, von Gruenigen VE, Schwartz AL, Medicine A (2010) American College of Sports Medicine roundtable on exercise guidelines for cancer survivors. Med Sci Sports Exerc 42(7):1409-1426. https://doi.org/10.1249/ MSS.0b013e3181e0c112

12. Tjonna AE, Lee SJ, Rognmo O, Stolen TO, Bye A, Haram PM, Loennechen JP, Al-Share QY, Skogvoll E, Slordahl SA, Kemi OJ, Najjar SM, Wisloff U (2008) Aerobic interval training versus continuous moderate exercise as a treatment for the metabolic syndrome: a pilot study. Circulation 118(4):346-354. https://doi. org/10.1161/circulationaha.108.772822

13. Wisloff U, Stoylen A, Loennechen JP, Bruvold M, Rognmo O, Haram PM, Tjonna AE, Helgerud J, Slordahl SA, Lee SJ, Videm V, Bye A, Smith GL, Najjar SM, Ellingsen O, Skjaerpe T (2007) Superior cardiovascular effect of aerobic interval training versus moderate continuous training in heart failure patients: a randomized study. Circulation 115(24):3086-3094. https://doi. org/10.1161/circulationaha.106.675041

14. Jaureguizar KV, Vicente-Campos D, Bautista LR, de la Pena CH, Gomez MJ, Rueda MJ, Fernandez Mahillo I (2016) Effect of highintensity interval versus continuous exercise training on functional capacity and quality of life in patients with coronary artery disease: a randomized clinical trial. J Cardiopulm Rehabil Prev 36(2):96-105. https://doi.org/10.1097/hcr.0000000000000156

15. Thum JS, Parsons G, Whittle T, Astorino TA (2017) High-intensity interval training elicits higher enjoyment than moderate intensity continuous exercise. PLoS ONE 12(1):e0166299. https://doi. org/10.1371/journal.pone.0166299

16. Ouerghi N, Selmi O, Ben Khalifa W, Ben Fradj MK, Feki M, Kaabachi N, Bouassida A (2016) Effect of high-intensity intermittent training program on mood state in overweight/obese young men. Iran J Public Health 45(7):951-952

17. Drigny J, Gremeaux V, Dupuy O, Gayda M, Bherer L, Juneau M, Nigam A (2014) Effect of interval training on cognitive functioning and cerebral oxygenation in obese patients: a pilot study. J Rehabil Med 46(10):1050-1054. https://doi. org/10.2340/16501977-1905

18. Saanijoki T, Tuominen L, Tuulari JJ, Nummenmaa L, Arponen E, Kalliokoski K, Hirvonen J (2017) Opioid release after highintensity interval training in healthy human subjects. Neuropsychopharmacology. https://doi.org/10.1038/npp.2017.148

19. Schmitt J, Lindner N, Reuss-Borst M, Holmberg HC, Sperlich B (2016) A 3-week multimodal intervention involving high-intensity interval training in female cancer survivors: a randomized controlled trial. Physiol Rep. https://doi.org/10.14814/phy2.12693

20. Dolan LB, Campbell K, Gelmon K, Neil-Sztramko S, Holmes D, McKenzie DC (2016) Interval versus continuous aerobic exercise training in breast cancer survivors: a pilot RCT. Support Care Cancer 24(1):119-127. https://doi.org/10.1007/s00520-015-2749-y

21. Schulz SVW, Laszlo R, Otto S, Prokopchuk D, Schumann U, Ebner F, Huober J, Steinacker JM (2017) Feasibility and effects of a combined adjuvant high-intensity interval/strength training in breast cancer patients: a single-center pilot study. Disabil Rehabil. https://doi.org/10.1080/09638288.2017.1300688

22. Green N, Wertz T, LaPorta Z, Mora A, Serbas J, Astorino TA (2017) Comparison of acute physiological and psychological responses between moderate intensity continuous exercise and three regimes of high intensity training. J Strength Cond Res. https://doi.org/10.1519/JSC.0000000000002154

23. Wengström Y, Bolam KA, Mijwel S, Sundberg CJ, Backman M, Browall M, Norrbom J, Rundqvist H (2017) Optitrain: a randomised controlled exercise trial for women with breast cancer undergoing chemotherapy. BMC Cancer 17(1):100. https://doi. org/10.1186/s12885-017-3079-x

24. Börjesson M, Urhausen A, Kouidi E, Dugmore D, Sharma S, Halle M, Heidbuchel H, Björnstad HH, Gielen S, Mezzani A, Corrado D, Pelliccia A, Vanhees L (2011) Cardiovascular evaluation of middle-aged/senior individuals engaged in leisure-time sport activities: position stand from the sections of exercise physiology and sports cardiology of the European Association of Cardiovascular Prevention and Rehabilitation. Eur J Cardiovasc Prev Rehabil 18(3):446-458. https://doi.org/10.1097/ HJR.0b013e32833bo969

25. Borg GA (1982) Psychophysical bases of perceived exertion. Med Sci Sports Exerc 14(5):377-381

26. Mayhew JL, Prinster JL, Ware JS, Zimmer DL, Arabas JR, Bemben MG (1995) Muscular endurance repetitions to predict bench press strength in men of different training levels. J Sports Med Phys Fitness 35(2): 108-113

27. Jakobsson S, Taft C, Östlund U, Ahlberg K (2013) Performance of the Swedish version of the Revised Piper Fatigue Scale. Eur J Oncol Nurs 17(6):808-813. https://doi.org/10.1016/j. ejon.2013.07.004

28. Piper BF, Dibble SL, Dodd MJ, Weiss MC, Slaughter RE, Paul SM (1998) The revised Piper Fatigue Scale: psychometric evaluation in women with breast cancer. Oncol Nurs Forum 25(4):677-684

29. Aaronson NK, Ahmedzai S, Bergman B, Bullinger M, Cull A, Duez NJ, Filiberti A, Flechtner H, Fleishman SB, de Haes JC et al (1993) The European Organization for Research and Treatment of Cancer QLQ-C30: a quality-of-life instrument for use in international clinical trials in oncology. J Natl Cancer Inst 85(5):365-376

30. Minton O, Stone P (2009) A systematic review of the scales used for the measurement of cancer-related fatigue (CRF). Ann Oncol 20(1):17-25. https://doi.org/10.1093/annonc/mdn537

31. Uwer L, Rotonda C, Guillemin F, Miny J, Kaminsky MC, Mercier M, Tournier-Rangeard L, Leonard I, Montcuquet P, Rauch P, Conroy T (2011) Responsiveness of EORTC QLQ-C30, QLQCR38 and FACT-C quality of life questionnaires in patients with colorectal cancer. Health Qual Life Outcomes 9:70. https://doi. org/10.1186/1477-7525-9-70 
32. Portenoy RK, Thaler HT, Kornblith AB, Lepore JM, FriedlanderKlar H, Kiyasu E, Sobel K, Coyle N, Kemeny N, Norton L et al (1994) The Memorial Symptom Assessment Scale: an instrument for the evaluation of symptom prevalence, characteristics and distress. Eur J Cancer (Oxford, England: 1990) 30A(9):1326-1336

33. Browall M, Sarenmalm EK, Nasic S, Wengstrom Y, GastonJohansson F (2012) Validity and reliability of the Swedish Version of the Memorial Symptom Assessment Scale (MSAS): an instrument for the evaluation of symptom prevalence, characteristics, and distress. J Pain Symptom Manage. https://doi.org/10.1016/j. jpainsymman.2012.07.023

34. Snyder CF, Blackford AL, Brahmer JR, Carducci MA, Pili R, Stearns V, Wolff AC, Dy SM, Wu AW (2010) Needs assessments can identify scores on HRQOL questionnaires that represent problems for patients: an illustration with the Supportive Care Needs Survey and the QLQ-C30. Qual Life Res 19(6):837-845. https:// doi.org/10.1007/s11136-010-9636-2

35. Aguilar-Farias N, Brown WJ, Peeters GM (2014) ActiGraph GT3X + cut-points for identifying sedentary behaviour in older adults in free-living environments. J Sci Med Sport 17(3):293299. https://doi.org/10.1016/j.jsams.2013.07.002

36. Morris SB (2007) Estimating effect sizes from pretest-posttestcontrol group designs. Organ Res Methods 11(2):364-386. https:// doi.org/10.1177/1094428106291059

37. Cohen J (1988) Statistical power analysis for the behavioral sciences, 2nd edn. Routledge, London

38. Rubin LH, Witkiewitz K, Andre JS, Reilly S (2007) Methods for handling missing data in the behavioral neurosciences: don't throw the baby rat out with the bath water. JUNE 5(2):A71-A77

39. van Waart H, Stuiver MM, van Harten WH, Geleijn E, Kieffer JM, Buffart LM, de Maaker-Berkhof M, Boven E, Schrama J, Geenen MM, Meerum Terwogt JM, van Bochove A, Lustig V, van den Heiligenberg SM, Smorenburg CH, Hellendoorn-van Vreeswijk JA, Sonke GS, Aaronson NK (2015) Effect of low-intensity physical activity and moderate- to high-intensity physical exercise during adjuvant chemotherapy on physical fitness, fatigue, and chemotherapy completion rates: results of the PACES Randomized Clinical Trial. J Clin Oncol 33(17):1918-1927. https:// doi.org/10.1200/jco.2014.59.1081

40. Mutrie N, Campbell AM, Whyte F, McConnachie A, Emslie C, Lee L, Kearney N, Walker A, Ritchie D (2007) Benefits of supervised group exercise programme for women being treated for early stage breast cancer: pragmatic randomised controlled trial. BMJ 334(7592):517. https://doi.org/10.1136/bmj.39094.648553.AE

41. Campbell A, Mutrie N, White F, McGuire F, Kearney N (2005) A pilot study of a supervised group exercise programme as a rehabilitation treatment for women with breast cancer receiving adjuvant treatment. Eur J Oncol Nurs 9(1):56-63. https://doi.org/10.1016/j. ejon.2004.03.007

42. Adamsen L, Quist M, Andersen C, Moller T, Herrstedt J, Kronborg D, Baadsgaard MT, Vistisen K, Midtgaard J, Christiansen B, Stage M, Kronborg MT, Rorth M (2009) Effect of a multimodal high intensity exercise intervention in cancer patients undergoing chemotherapy: randomised controlled trial. BMJ 339:b3410. https://doi.org/10.1136/bmj.b3410

43. McAuley E, Blissmer B, Katula J, Duncan TE, Mihalko SL (2000) Physical activity, self-esteem, and self-efficacy relationships in older adults: a randomized controlled trial. Ann Behav Med 22(2):131-139
44. Penninx BW, Rejeski WJ, Pandya J, Miller ME, Di Bari M, Applegate WB, Pahor M (2002) Exercise and depressive symptoms: a comparison of aerobic and resistance exercise effects on emotional and physical function in older persons with high and low depressive symptomatology. J Gerontol Ser B 57(2):P124-P132

45. Furmaniak AC, Menig M, Markes MH (2016) Exercise for women receiving adjuvant therapy for breast cancer. Cochrane Database Syst Rev. https://doi.org/10.1002/14651858.cd005001.pub3

46. Schmidt T, Weisser B, Durkop J, Jonat W, Van Mackelenbergh M, Rocken C, Mundhenke C (2015) Comparing endurance and resistance training with standard care during chemotherapy for patients with primary breast cancer. Anticancer Res 35(10):5623-5629

47. Travier N, Velthuis MJ, Steins Bisschop CN, van den Buijs B, Monninkhof EM, Backx F, Los M, Erdkamp F, Bloemendal HJ, Rodenhuis C, de Roos MA, Verhaar M, ten Bokkel Huinink D, van der Wall E, Peeters PH, May AM (2015) Effects of an 18-week exercise programme started early during breast cancer treatment: a randomised controlled trial. BMC Med 13:121. https://doi.org/10.1186/s12916-015-0362-z

48. Lyon DE, Cohen R, Chen H, Kelly DL, Starkweather A, Ahn HC, Jackson-Cook CK (2016) The relationship of cognitive performance to concurrent symptoms, cancer- and cancer-treatmentrelated variables in women with early-stage breast cancer: a 2-year longitudinal study. J Cancer Res Clin Oncol 142(7):1461-1474. https://doi.org/10.1007/s00432-016-2163-y

49. Saibil S, Fitzgerald B, Freedman OC, Amir E, Napolskikh J, Salvo N, Dranitsaris G, Clemons M (2010) Incidence of taxane-induced pain and distress in patients receiving chemotherapy for earlystage breast cancer: a retrospective, outcomes-based survey. Curr Oncol 17(4):42-47

50. van Helmond N, Steegers MA, Filippini-de Moor GP, Vissers KC, Wilder-Smith OH (2016) Hyperalgesia and persistent pain after breast cancer surgery: a prospective randomized controlled trial with perioperative COX-2 inhibition. PLOS ONE 11(12):e0166601. https://doi.org/10.1371/journal.pone.0166601

51. Stasi R, Abriani L, Beccaglia P, Terzoli E, Amadori S (2003) Cancer-related fatigue: evolving concepts in evaluation and treatment. Cancer 98(9):1786-1801. https://doi.org/10.1002/cncr.11742

52. Courneya KS, Segal RJ, McKenzie DC, Dong H, Gelmon K, Friedenreich CM, Yasui Y, Reid RD, Crawford JJ, Mackey JR (2014) Effects of exercise during adjuvant chemotherapy on breast cancer outcomes. Med Sci Sports Exerc 46(9):1744-1751. https:// doi.org/10.1249/mss.0000000000000297

53. Wu HS, Harden JK (2015) Symptom burden and quality of life in survivorship: a review of the literature. Cancer Nurs 38(1):E29E54. https://doi.org/10.1097/ncc.0000000000000135

54. Taaffe DR, Newton RU, Spry N, Joseph D, Chambers SK, Gardiner RA, Wall BA, Cormie P, Bolam KA, Galvao DA (2017) Effects of different exercise modalities on fatigue in prostate cancer patients undergoing androgen deprivation therapy: a year-long randomised controlled trial. Eur Urol 72(2):293-299. https://doi. org/10.1016/j.eururo.2017.02.019

55. Carayol M, Delpierre C, Bernard P, Ninot G (2015) Population-, intervention- and methodology-related characteristics of clinical trials impact exercise efficacy during adjuvant therapy for breast cancer: a meta-regression analysis. Psycho-oncology 24(7):737747. https://doi.org/10.1002/pon.3727 